\title{
An Analytical Study of Ozone Feedbacks on Kelvin and Rossby-Gravity Waves: Effects on the QBO
}

\author{
Eugene C. Cordero* and Terrence R. Nathan \\ Atmospheric Science Program, Department of Land, Air and Water Resources, University of California, Davis, \\ Davis, California
}

ROBERT S. ECHOLS

Department of Physics, University of California, Santa Cruz, Santa Cruz, California

(Manuscript received 13 May 1996, in final form 22 July 1997)

ABSTRACT

\begin{abstract}
An equatorial beta-plane model of the middle atmosphere is used to analytically examine the effects of radiative cooling and ozone heating on the spatial and temporal evolution of the quasi-biennial oscillation (QBO). Under the assumption that the diabatic heating is weak and the background fields of wind, temperature, and ozone are slowly varying, a perturbation analysis yields expressions describing the vertical spatial modulation of Kelvin and Rossby-gravity waves in the presence of ozone. These expressions show that wave-induced changes in the diabatic heating arising from the advection of basic-state ozone reduce the local radiative damping rate by up to $15 \%$ below $35 \mathrm{~km}$. In a one-dimensional model of the QBO, eddy ozone heating increases the amplitude of the zonal wind QBO by 1-2 $\mathrm{m} \mathrm{s}^{-1}$ and increases the oscillation period by about two months. The significance of these results to the observed $\mathrm{QBO}$ is discussed.
\end{abstract}

\section{Introduction}

The quasi-biennial oscillation (QBO) in zonal wind was first observed independently by Reed et al. (1961) and Veryard and Ebdon (1961) and has since been studied extensively (Nastrom and Belmont 1975; Coy 1979; Plumb 1984; Dunkerton 1985; Andrews et al. 1987). The QBO is confined to equatorial latitudes and is characterized by alternating easterly and westerly winds that oscillate with a variable period ranging from 22 to 34 months. The zonal wind reversal initially occurs at higher levels in the stratosphere (above $30 \mathrm{~km}$ ) and gradually descends at a rate of approximately one kilometer per month. Maintenance of thermal wind balance explains the observed temperature QBO, which has a maximum amplitude of approximately $3 \mathrm{~K}$ in the lower equatorial stratosphere (Plumb 1984; Andrews et al. 1987). The secondary circulation that maintains the temperature structure of the QBO is responsible for the tropical ozone QBO (Reed 1964; Plumb and Bell 1982; Hasebe 1984; Ling and London 1986).

\footnotetext{
* Current affiliation: Universities Space Research Association, NASA/Goddard Space Flight Center, Greenbelt, Maryland.

Corresponding author address: Dr. Eugene C. Cordero, NASA/ GSFC, Code 916, Greenbelt, MD 20771.

E-mail: cordero@polska.gsfc.nasa.gov
}

The commonly accepted theory explaining the generation and maintenance of the zonal wind QBO asserts that damped vertically propagating equatorial waves are the source of momentum driving the zonal mean flow (Lindzen and Holton 1968; Holton and Lindzen 1972). Holton and Lindzen (hereafter HL) used a one-dimensional (1D) model to demonstrate how thermal and mechanical damping of forced, vertically propagating equatorial Kelvin and Rossby-gravity waves can produce a QBO-like circulation. Since HL's study, more realistic two and three-dimensional mechanistic models have verified the importance of wave damping in driving the QBO (Plumb and Bell 1982; Dunkerton 1985; Takahashi 1987; Takahashi and Boville 1992). However, these models require unrealistically high wave amplitudes in order to produce a QBO consistant with observations.

Studies that have investigated the existence of alternate momentum sources large enough to drive the QBO have thus far been inconclusive. For example, Takahashi and Holton (1991) found that the inability of Rossbygravity waves to provide sufficient momentum to drive the easterly phase of the QBO cannot be resolved by either Rossby waves or a single westward propagating gravity wave. Their conclusions, along with those of more recent studies using general circulation models (GCMs), are that smaller-scale gravity waves may be important for driving the QBO (Hayashi and Golder 
1994; Takahashi 1996). However, the continuing difficulties in obtaining a realistic QBO in GCMs suggest that other mechanisms may also play an important role in the QBO.

Central to understanding the generation and maintenance of the QBO is the process of wave damping, a mechanism by which vertically propagating waves deposit their momentum flux to the zonal mean flow. Models of the QBO have verified the importance of Newtonian cooling (NC) on wave damping but have generally neglected the effects of ozone heating on the waves.

Recent studies have indeed shown that ozone heating can have an important effect on wave motions in the atmosphere. For example, Zhu and Holton (1986) found that the diabatic heating due to vertical ozone advection and ozone photochemistry can increase the local NC rate by as much as $50 \%$ for forced inertio-gravity waves. Nathan (1989), Nathan and Li (1991), and Nathan et al. (1994) showed that radiative-photochemical feedbacks involving ozone can destabilize free Rossby waves in midlatitudes. More recently, Echols and Nathan (1996) demonstrated that ozone-dynamics interactions can change the divergence of Eliassen-Palm (EP) flux for forced equatorial Kelvin waves by as much as $25 \%$ in the stratosphere. Because Kelvin waves are at least partially responsible for the westerly phase of the QBO, the results of Echols and Nathan indicate that ozonedynamics interactions may play a more important role in influencing the circulation of the equatorial lower stratosphere than previously thought.

Improving our understanding of the role ozone heating plays in equatorial wave dynamics may provide answers to important questions surrounding the QBO. For example, what influence do radiative-photochemical feedbacks have on the spatial and temporal properties of forced, equatorial Kelvin and Rossby-gravity waves? How does the diabatic heating arising from ozone-dynamics interactions in the equatorial stratosphere affect wave damping, a process vital to driving the zonal mean flow? How important is the interaction among the eddy fields of ozone, wind, and temperature in the production and maintenance of the zonal wind QBO?

Motivated by these questions, we investigate two aspects of the QBO problem that have not been fully addressed: First, we examine the role ozone heating plays in affecting the spatial modulation of forced Rossby-gravity waves; second, we examine how the eddy feedbacks among radiational cooling, ozone heating, and dynamics influence the zonal wind QBO. Thus, in contrast to recent work by Hasebe (1994), who examined how the heating due to the ozone QBO influences the zonal wind QBO, our focus is on how the eddy ozone heating interacts with the wave fields, which in turn drive the zonal mean circulation via wave damping.

The paper is organized as follows: In section 2 the model and basic states are described. Section 3 contains the analytical development and solutions for both the
Kelvin and Rossby gravity waves. A description of our 1D model and results are presented in section 4, followed by a discussion of the relevance of this study to the observed QBO. The summary and conclusions are presented in section 5 .

\section{Equatorial $\boldsymbol{\beta}$-plane model}

\section{a. Governing equations and boundary conditions}

The model atmosphere is governed by linearized primitive equations on an equatorial $\beta$ plane (Andrews et al. 1987; Echols and Nathan 1996). These equations describe, respectively, conservation of zonal and meridional momentum, conservation of mass, hydrostatic balance, thermodynamic energy, and ozone volume mixing ratio, which in log-pressure coordinates can be written as

$$
\begin{aligned}
\left(\frac{\partial}{\partial t}+\bar{u} \frac{\partial}{\partial x}\right) u^{\prime}+w^{\prime} \frac{\partial \bar{u}}{\partial z}-\beta y v^{\prime} & =-\frac{\partial \Phi^{\prime}}{\partial x} \\
\left(\frac{\partial}{\partial t}+\bar{u} \frac{\partial}{\partial x}\right) v^{\prime}+\beta y u^{\prime} & =-\frac{\partial \Phi^{\prime}}{\partial y} \\
\frac{\partial u^{\prime}}{\partial x}+\frac{\partial v^{\prime}}{\partial y}+\frac{1}{\rho} \frac{\partial\left(\rho w^{\prime}\right)}{\partial z} & =0 \\
\left(\frac{\partial}{\partial t}+\bar{u} \frac{\partial}{\partial x}\right) T^{\prime}+v^{\prime} \frac{\partial \bar{T}}{\partial y}+\frac{H}{R} N^{2} w^{\prime} & =-\alpha T^{\prime}+\frac{H}{R} A \gamma^{\prime} \\
\left(\frac{\partial}{\partial t}+\bar{u} \frac{\partial}{\partial x}\right) \gamma^{\prime}+v^{\prime} \frac{\partial \bar{\gamma}}{\partial y}+w^{\prime} \frac{\partial \bar{\gamma}}{\partial z} & =-B \gamma^{\prime}-\frac{R}{H} C T^{\prime}
\end{aligned}
$$

The symbols in (1)-(6) are defined in Table 1 where zonal mean quantities are denoted by an overbar and perturbations from the zonal mean are denoted by a prime.

The parameterizations for the ozone heating/photochemical coefficients in (5) and (6) are similar to previous studies of ozone dynamics interactions (Hartmann and Garcia 1979; Zhu and Holton 1986; Nathan and Li 1991; Echols and Nathan 1996). Briefly, $A$ is the ozone heating coefficient, which is based on the diurnal averaging procedure of Cogley and Borucki (1976) to calculate the absorption of solar radiation and photodissociation rates per ozone molecule (Nathan and $\mathrm{Li}$ 1991). The effects of multiple scattering and ground reflection are parameterized as in Strobel (1978). In (6) $B$ and $C$ are parameterizations of the photochemical response of ozone to dynamically induced perturbations in ozone and temperature, respectively. Catalytic loss processes involving odd nitrogen, odd chlorine, and odd hydrogen are included and are based on Stolarski and Douglass (1985). The vertical distributions of $A, B$, and $C$ at the equator are portrayed in Fig. 1. Inclusion of 
TABLE 1. List of symbols.

\begin{tabular}{ll}
\hline \hline$x, y, z=-H \ln \left(p / p_{0}\right)$ & Eastward, northward, and vertical direction \\
$p_{0}$ & Sea level reference pressure $(=1000 \mathrm{hPa})$ \\
$H$ & Scale height $(=6 \mathrm{~km})$ \\
$\rho(z)=\rho_{0} \exp (-z / H)$ & Basic-state density \\
$\rho_{0}$ & Global mean sea level density $\left(1 \mathrm{~kg} \mathrm{~m}^{-3}\right)$ \\
$\underline{\beta}$ & Northward gradient of the Coriolis parameter evaluated at the equator $\left(=2.29 \times 10^{-11} \mathrm{~m}^{-1} \mathrm{~s}^{-1}\right)$ \\
$u(z), \bar{T}(y, z), \bar{\gamma}(y, z)$ & Basic-state zonal mean wind, temperature, and ozone volume mixing ratio \\
$N^{2}$ & Brünt-Väisälä frequency squared \\
$c$ & Wave phase speed \\
$k$ & Zonal wavenumber; $s=k a_{\mathrm{e}}$, where $\mathrm{s}=1,2,3, \cdots$ and $a_{\mathrm{e}}$ is the earth's radius \\
$u^{\prime}(x, y, z, t), v^{\prime}(x, y, z, t)$ & Perturbation zonal and meridional wind components \\
$w^{\prime}(x, y, z, t)$ & Perturbation vertical wind component \\
$T^{\prime}(x, y, z, t)$ & Perturbation temperature \\
$\Phi^{\prime}(x, y, z, t)$ & Perturbation geopotential \\
$\gamma^{\prime}(x, y, z, t)$ & Perturbation ozone volume mixing ratio \\
$a(z)$ & Radiative cooling coefficient \\
$A(z ; \bar{T}, \bar{\gamma})$ & Ozone heating coefficient \\
$B(z ; T, \gamma), C(z ; \bar{T}, \bar{\gamma})$ & Radiative-photochemical coefficients in the ozone continuity equation \\
\hline
\end{tabular}

the shielding effect, which accounts for the influence of perturbations in ozone above a given height, are neglected because of the relatively short vertical scale of the Kelvin and Rossby-gravity waves considered here (Echols and Nathan 1996).

Because the equatorial waves considered here have relatively short vertical wavelengths, the thermal relaxation rate $\alpha$, which appears in the Newtonian cooling term in (5), is calculated based on the scale-dependent radiative relaxation scheme of Fels (1982) and is shown in Fig. 2. For the Kelvin wave we have chosen a vertical wavelength of $12 \mathrm{~km}$, while for the Rossby-gravity wave we have chosen a vertical wavelength of $8 \mathrm{~km}$ (Andrews et al. 1987).

The temperature and ozone fields are directly coupled by the diabatic heating term in the thermodynamic energy equation (5) and the ozone production/destruction terms in the ozone continuity equation (6). Wave-induced ozone perturbations, $\gamma^{\prime}$, alter the diabatic heating rate per unit mass $\left(Q^{\prime}=H R^{-1} A \gamma^{\prime}-\alpha T^{\prime}\right)$ and thus temperature perturbations, $T^{\prime}$, which in turn alter the ozone production/destruction $\left(S^{\prime}=-B \gamma^{\prime}-R H^{-1} T^{\prime}\right)$ and thus ozone perturbations.

Our numerical analysis described in section 4 uses the following boundary conditions. At the lower boundary the amplitude, period, and wavelength of both the Kelvin and Rossby-gravity wave is specified (HL; Dunkerton 1979). A radiation condition is applied at the upper boundary; that is, we require that the vertical energy flux be bounded and directed toward infinity as $z \rightarrow \infty$. In the meridional direction wave solutions are equatorially trapped (e.g., $\Phi \rightarrow 0$ as $y \rightarrow \infty$ ) and in the zonal direction we assume the solutions are periodic.

\section{b. Basic states}

Although seasonal variations exist in the equatorial distributions of zonal mean temperature and zonal mean ozone, these variations are relatively small and thus have little effect on the wave responses or their feedbacks with the zonal mean flow. Therefore, we choose for presentation the results obtained from a basic state representative of the September equinox at the equator.

Figure 3 displays the zonal mean temperature distribution, $\bar{T}(z)$, at the equator. This distribution is calculated from data compiled by Fleming et al. (1988), who synthesized monthly mean temperature fields using both ground-based and satellite data.

To facilitate our analytical analysis, we represent the zonal mean ozone distribution in the Tropics as

$$
\bar{\gamma}(y, z)=\frac{a y^{2}}{2} \hat{\bar{\gamma}}_{y}(z)+\bar{\gamma}(0, z),
$$

where $y$ is the meridional distance from the equator, $\hat{\bar{\gamma}}_{y}(z)$ is the height-dependent meridional gradient of basic-state ozone taken at $15^{\circ} \mathrm{N}$, and $a=1.67 \times 10^{-6} \mathrm{~m}^{-1}$ represents the inverse of the distance from the equator to $15^{\circ}$ latitude. The zonal mean ozone distribution given by (7) resembles observations in the tropical stratosphere while explicitly accounting for the approximate linear relationship between $y$ and $\bar{\gamma}_{y}$ in the Tropics (e.g., $\bar{\gamma}_{y}=a y \hat{\bar{\gamma}}_{y}$ ). Figure $4 \mathrm{a}$ displays the basic-state ozone volume mixing ratio distribution, $\bar{\gamma}(z)$, at the equator. This distribution is based on values from Keating and Young (1985) above $30 \mathrm{mb}$ and McPeters et al. (1984) below $30 \mathrm{mb}$. A cubic spline algorithm is used to interpolate the zonal mean ozone data into the desired resolution; the vertical and meridional gradients in basic-state ozone, $\bar{\gamma}_{z}$ and $\bar{\gamma}_{y}$, shown in Figs. $4 \mathrm{~b}$ and $4 \mathrm{c}$, are computed using second-order finite differences.

In the analytical section, two basic-state zonal wind profiles representative of the two phases of the QBO are considered:

$$
\bar{u}(z)= \pm 20 \tanh \left[\left(z-z_{0}\right) / 3\right], \quad z_{0}=27 \mathrm{~km},
$$

where $z_{0}$ corresponds to the level of maximum wind shear. Profile 1 ( + sign) corresponds to the descending westerly phase of the QBO and profile 2 ( - sign) corresponds to the descending easterly phase of the QBO. These wind profiles are displayed in Fig. 5. 

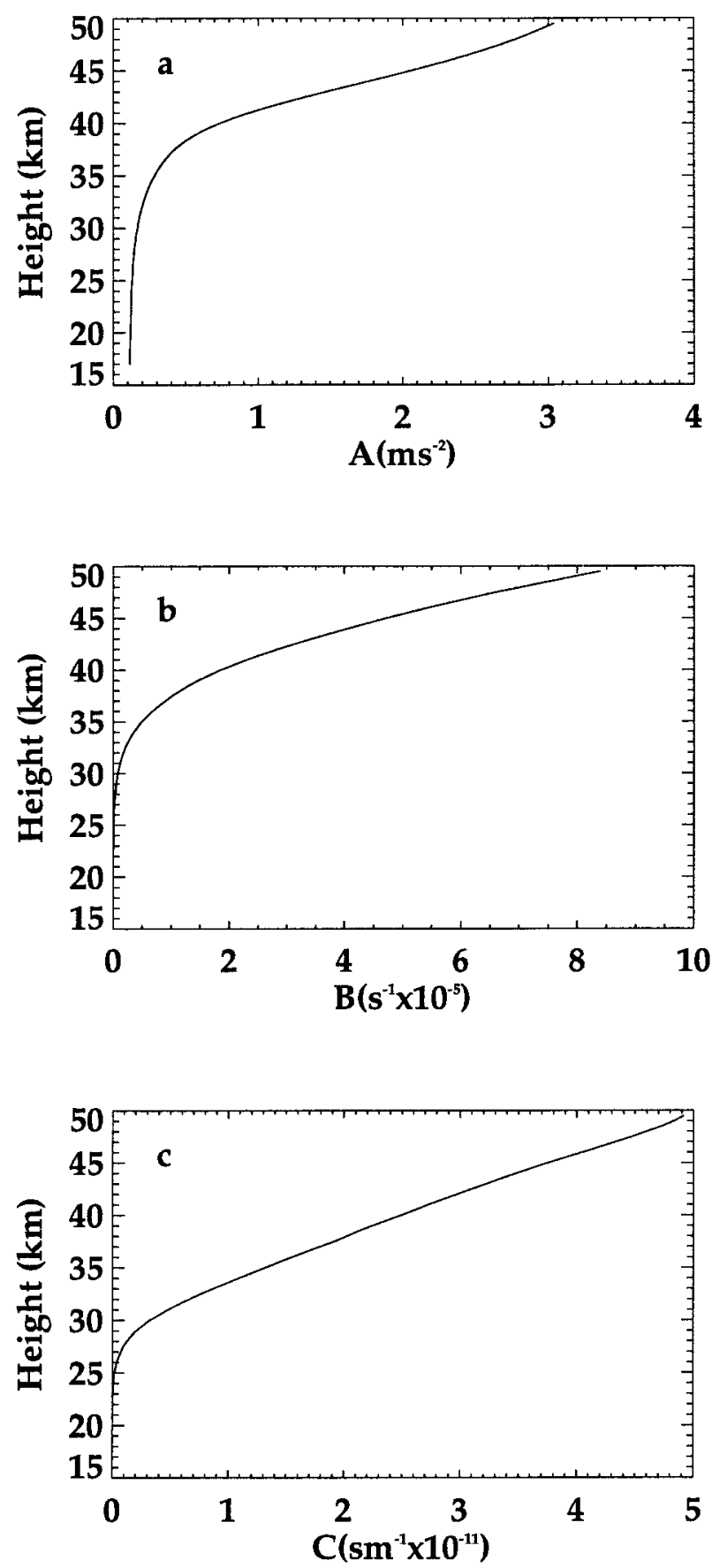

FIG. 1. Distribution of the (a) local ozone heating coefficient $A$, (b) ozone damping coefficient $B$, and (c) ozone-temperature coupling coefficient $C$.

\section{Analytical analysis}

In this section we derive analytical expressions for the amplitude and phase of forced, equatorial Kelvin and Rossby-gravity waves in the presence of radiative cooling and ozone heating. We employ the perturbation analysis technique described in Andrews and McIntyre (1976). Details of the mathematical development are

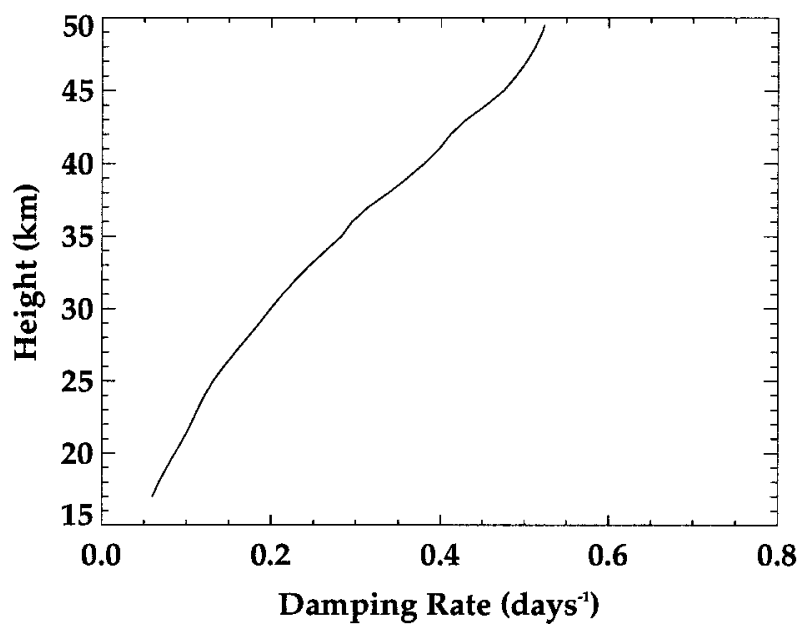

FIG. 2. Scale-dependent radiative cooling coefficient, $\alpha\left(\right.$ day $\left.^{-1}\right)$.

provided in appendix A. Briefly, the analysis hinges on the assumption that the background basic state is slowly varying in the vertical and that the diabatic heating is weak. To account for the "slow" vertical spatial modulation of the wave fields, we introduce a long vertical scale, $\zeta=\mu z$, where $\mu \ll 1$ is nondimensional, and transform the vertical differential operator as $\partial / \partial z \Rightarrow \partial /$ $\partial z+\mu \partial / \partial \zeta^{1}$. We also assume that diabatic processes resulting from radiative cooling and ozone heating are small; that is, $Q^{\prime} \Rightarrow \mu Q^{\prime}$. With the above reordering of the vertical scale and diabatic heating, the basic-state fields of wind, temperature, and ozone are chosen of the form $\bar{u}=\bar{u}(\zeta), \bar{T}=\bar{T}(y, \zeta), \bar{\gamma}=\bar{\gamma}(y, \zeta)$.

All dependent variables are expanded in a perturbation series of the form

$$
\begin{aligned}
& g^{\prime}(x, y, z, \zeta, t ; \mu)=e^{z / 2 H} \operatorname{Re}\left\{\left[\hat{g}_{0}(y, \zeta)+\mu \hat{g}_{1}(y, \zeta)\right.\right. \\
& \left.+\mu^{2} \hat{g}_{2}(y, \zeta)+\cdots\right] \\
& \left.\times \operatorname{expi}\left(k x-\sigma t+\mu^{-1} \phi(\zeta)\right)\right\} \text {, }
\end{aligned}
$$

where $\hat{g}_{n}(y, \zeta)$ is the wave structure function, $k$ is the zonal wavenumber, and $\sigma$ is the ground-based frequency. Insertion of (9) into (1)-(6) yields a sequence of problems in powers of $\mu$; the lowest (zeroth) order system of equations yield the classic solutions for the Kelvin and Rossby-gravity wave (Lindzen 1971), namely,

\footnotetext{
${ }^{1}$ Andrews and McIntyre's (1976) analytical study considers the possibility of wave transience and wave dissipation, thus necessitating the introduction of long time and space scales to account for both the temporal and spatial modulation of the wave fields. In contrast, we consider only the effects of wave dissipation, that is, we examine the diabatic effects of ozone heating and radiative cooling on the vertical spatial modulation of forced waves of fixed frequency; thus we only need to introduce a long vertical scale.
} 


$$
\begin{aligned}
& \left\{u_{0}, v_{0}, w_{0}, T_{0}, \Phi_{0}\right\} \\
& =a_{\mathrm{K}}(\zeta)\left\{1,0, \frac{\omega}{N},-i \frac{H N}{R}, \frac{\omega}{k}\right\} \exp \left(\frac{-\beta\left|m_{\mathrm{K}}\right| y^{2}}{2 N}\right), \\
& \left\{u_{0}, v_{0}, w_{0}, T_{0}, \Phi_{0}\right\} \\
& =a_{\mathrm{RG}}(\zeta)\left\{\frac{i\left|m_{\mathrm{RG}}\right| \omega y}{N}, 1, \frac{i\left|m_{\mathrm{RG}}\right| \omega^{2} y}{N^{2}}, \frac{\left|m_{\mathrm{RG}}\right| H \omega y}{R},\right. \\
& i \omega y\} \exp \left(\frac{-\beta\left|m_{\mathrm{RG}}\right| y^{2}}{2 N}\right),
\end{aligned}
$$

where $\omega=\omega(\zeta)=\sigma-k \bar{u}(\zeta)$ and the subscripts K and RG denote the Kelvin and Rossby-gravity waves, respectively. The wave amplitudes $a_{\mathrm{K}}(\zeta)$ and $a_{\mathrm{RG}}(\zeta)$ are thus far unknown and are determined at $O(\mu)$.

At $O(\mu)$ the diabatic effects due to $\mathrm{NC}$ and ozone heating along with the slow vertical variations enter as forcings on the linear operator. In order that the expansion (9) remain valid, these forcings must be orthogonal to the homogeneous adjoint solution. This requirement yields the sought-after expressions for the amplitudes of the Kelvin wave (denoted by $j=0$ ) and Rossbygravity wave (denoted by $j=1$ ):

$$
a_{j}(\zeta)=\frac{\text { const }}{|\omega|^{3 / 4}|\omega|^{3 j / 4}}[N(\beta+\omega k)]^{\mathrm{j} / 4} \exp \left(i \int m_{j}(\zeta) d \zeta\right),
$$

where the local vertical wavenumber is defined as

$$
m_{j}(\zeta)=m_{r, j}(\zeta)+i m_{i, j}(\zeta) .
$$

The subscripts $r$ and $i$ denote real and imaginary components. The real component of the vertical wavenumber, $m_{r}$, can be approximated by the standard dispersion relation for Kelvin and Rossby-gravity waves (see appendix $\mathrm{B}$ ); the imaginary component of the vertical wavenumber, $m_{i}$, is

$$
\begin{aligned}
m_{i, j} & =-\frac{m_{r, j}}{2 \omega}\left[\alpha+\frac{1}{\left(B^{2}+\omega^{2}\right)}\right. \\
& \left.\times\left\{\begin{array}{ll}
A B C-\frac{A \bar{\gamma}_{z} \omega^{2}}{N^{2}}-\delta_{j, 1} & \frac{A a \hat{\bar{\gamma}}_{y} B \omega}{N(\beta+\omega k)} \\
\underbrace{}_{\mathrm{I}}
\end{array}\right\}\right],
\end{aligned}
$$

where $\delta_{j, 1}$ is the Kronecker delta function, so that term III only applies to the Rossby-gravity wave. Equation (14) explicitly describes how ozone feedbacks modify the amplitude of the Kelvin and Rossby-gravity waves in the presence of both longwave radiational cooling and ozone heating. If ozone feedbacks are neglected, corresponding to $A=B=C=0$, (14) is equivalent

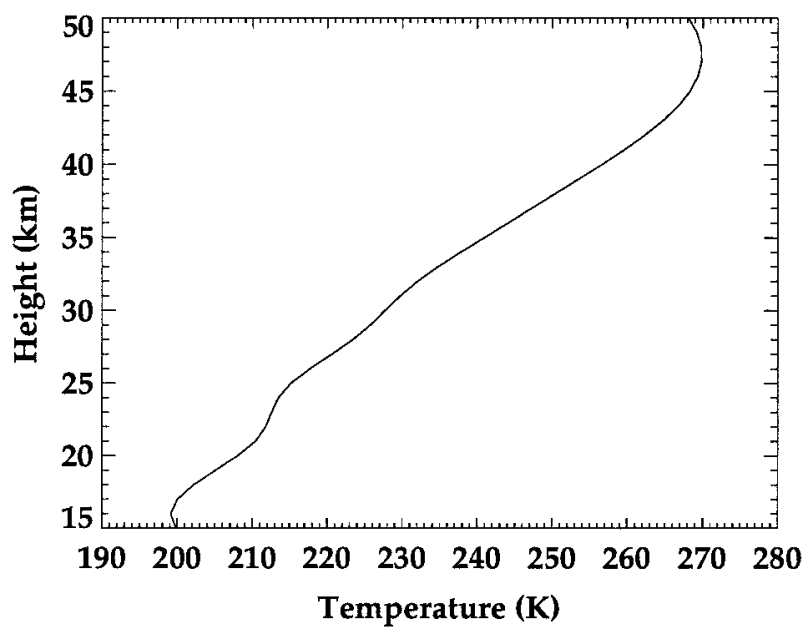

FIG. 3. Basic-state temperature $(\mathrm{K})$ at the equator for September.

to the damping rates obtained by Holton and Lindzen (1972).

\section{a. Rossby-gravity wave analysis}

Equation (14) reveals that the $\mathrm{NC}$ damping rate $\alpha$ is modified by three ozone heating effects: ozone photochemistry (I), vertical ozone advection (II), and meridional ozone advection (III). Depending upon the sign and relative magnitude of these terms, the (local) spatial damping rate of the wave is either increased or decreased.

Term I represents ozone photochemical heating, which in the lower stratosphere is one to two orders of magnitude smaller than terms II and III. In sharp contrast II and III, which represent vertical and meridional ozone advection, have significant magnitudes in the lower and midstratosphere.

Next we numerically compare the relative magnitude of the NC coefficient $(\alpha)$ with the ozone heating terms (I, II, and III) for the Rossby-gravity wave $(c=-30$ $\left.\mathrm{m} \mathrm{s}^{-1}, s=4\right)$. Figure 6 shows the relative contributions of I, II, and III to the total damping rate for zonal wind profiles 1 and 2. For descending westerlies (profile 1) below $30 \mathrm{~km}$, vertical ozone advection (II) reduces the local damping rate due to $\mathrm{NC}$ alone by up to $17 \%$. Above $35 \mathrm{~km}$, where $\bar{\gamma}_{z}<0$, the diabatic heating effects due to ozone advection are reversed, resulting in local damping times that are up to $20 \%$ larger. Meridional ozone advection (III), which has a negligible influence below $27 \mathrm{~km}$, only accounts for a slight increase in the damping times between 31 and $35 \mathrm{~km}$. Above $37 \mathrm{~km}$, $\bar{\gamma}_{y}>0, \omega<0$, and $(\beta-\omega k)>0$ so that meridional ozone advection enhances the damping rates by up to $15 \%$. Term I, which represents ozone photochemical processes, has little influence below $35 \mathrm{~km}$; above 40 $\mathrm{km}$ I increases the damping rates by over $25 \%$.

For descending easterlies (profile 2), the contributions from terms I-III are generally similar to their contri- 

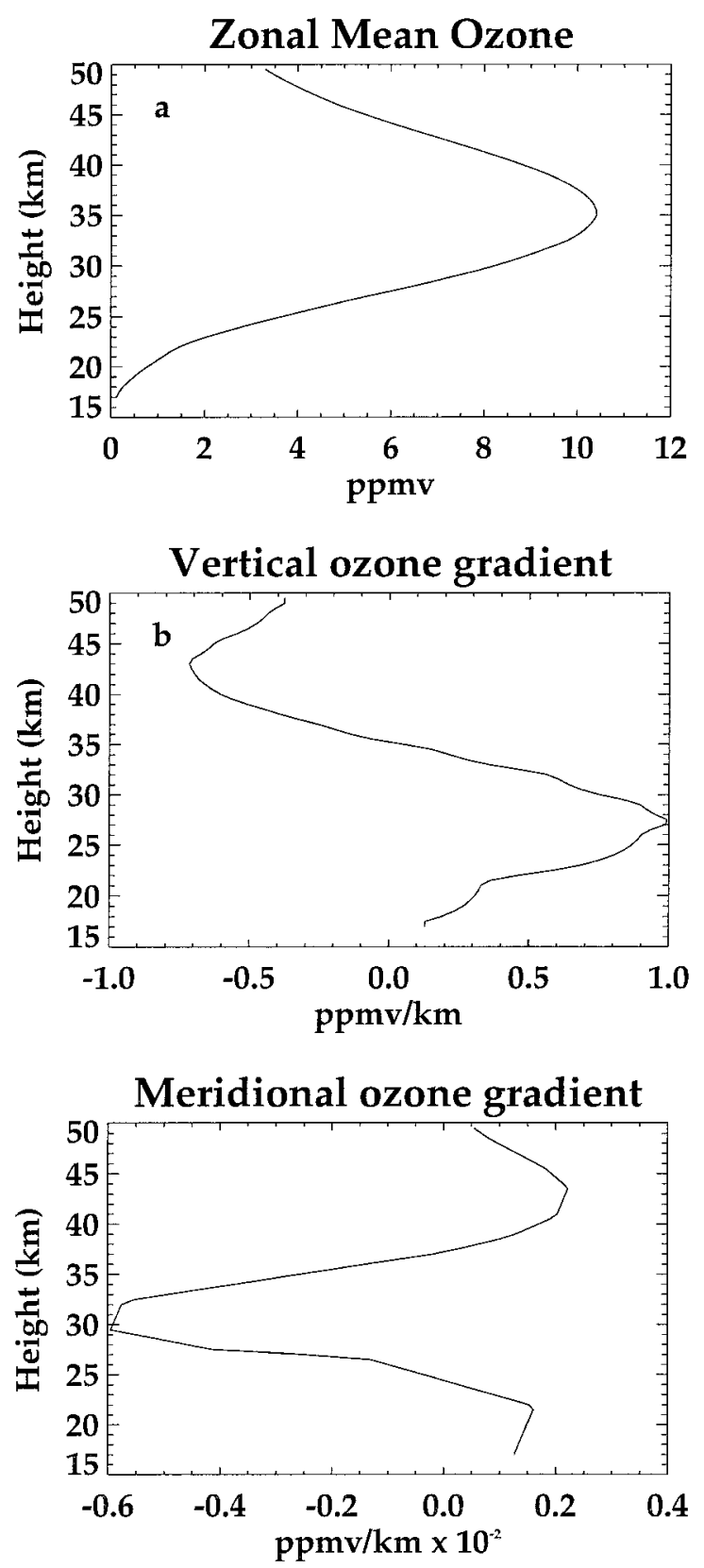

FIG. 4. Basic-state distribution of (a) ozone mixing ratio $\bar{\gamma}$ at the equator, (b) vertical gradient of basic-state ozone $\bar{\gamma}_{z}$ at the equator, and (c) meridional gradient of basic state ozone $\bar{\gamma}_{y}$ at $15^{\circ} \mathrm{N}$ for September.

bution to the case of descending westerlies (profile 1). However, there are some important differences. Below $30 \mathrm{~km}$, the ratio of photochemical to advective timescales is $\tau \equiv B / \omega \approx 10^{-2}$. Terms II and III are therefore only weakly dependent on $\omega$ below $30 \mathrm{~km}$, so the zonal wind profile has little effect on the damping rates. However, above $30 \mathrm{~km}, \tau^{2} \approx O(1)$ so that II and III are more dependent on $\omega$. Therefore, because $\omega$ is smaller for

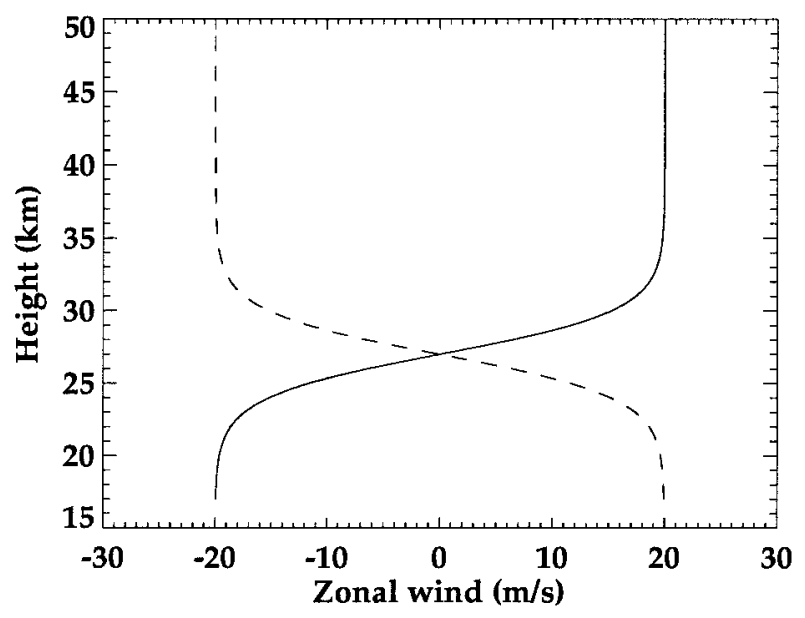

FIG. 5. Zonal mean wind profiles typical of the descending westerlies of the QBO (solid line: profile 1) and the descending easterlies of the $\mathrm{QBO}$ (dashed line: profile 2).

wind profile 2 than for wind profile 1, terms II and III make smaller contributions to the damping rate.

We explain the above results as follows. Below 30 $\mathrm{km}$, where dynamical timescales (10-50 days) are much shorter than photochemical timescales (100-1000 days) and the vertical gradient of basic-state ozone is positive $\left(\bar{\gamma}_{z}>0\right)$, perturbative vertical motions transport ozonepoor air upward and ozone-rich air downward, resulting in a net cooling above a net warming. This results in a reduction of the static stability and thus a decrease in the damping rate. In contrast, above $35 \mathrm{~km}$, where $\bar{\gamma}_{z}$ $<0$, vertical ozone advection has a stabilizing effect on wave amplitudes. Meridional ozone advection can also increase or decrease the damping rate in a similar manner, although the magnitude of these changes below $30 \mathrm{~km}$ is negligible. Above $35 \mathrm{~km}$ ozone is dominated by photochemical processes and thus wave-induced temperature perturbations are anticorrelated with perturbations in ozone due to temperature-dependent ozone photochemistry. A warm temperature anomaly results in less ozone and therefore, less heating through ozone's absorption of solar ultraviolet radiation. This processtermed photochemically accelerated cooling - produces strong thermal damping (Strobel 1977) and augments the thermal damping due to vertical ozone advection.

\section{b. Kelvin wave analysis}

The effects of ozone heating on the spatial modulation of forced equatorial Kelvin waves $\left(c=30 \mathrm{~m} \mathrm{~s}^{-1}, s=\right.$ 1) has been treated in detail by Echols and Nathan (1996). Although their study uses a slightly different analytical analysis, both approaches yield similar results for the lower stratosphere. Thus, we only briefly summarize the results for the Kelvin wave in order to ease comparison with the Rossby-gravity wave results given in section $3 \mathrm{c}$.

Inspection of (14) reveals that the functional forms 

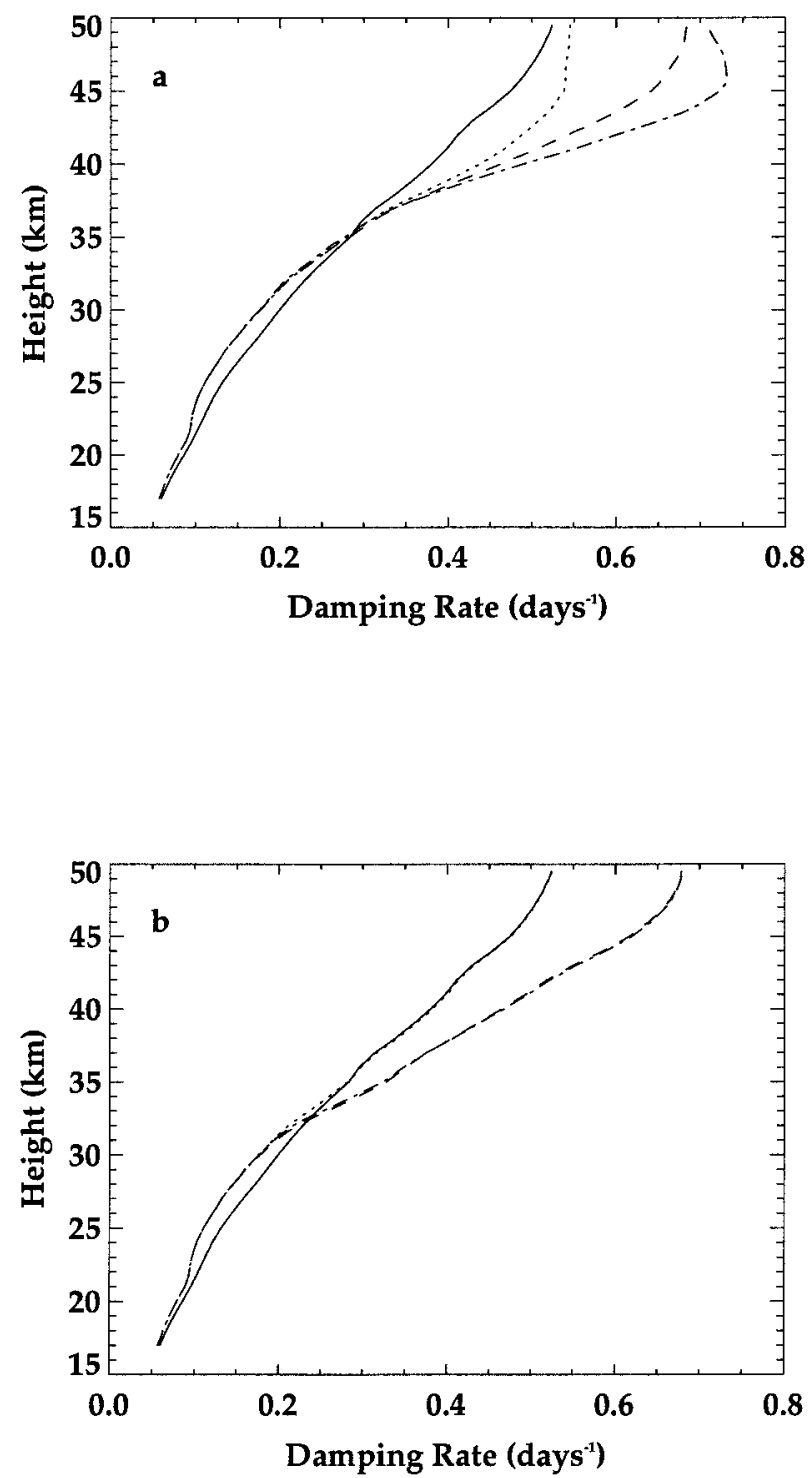

FIG. 6. Comparison of the radiative damping rate ( $\alpha$ : solid), radiative damping and vertical ozone advection ( $\alpha+$ term II: dotted), radiative damping, vertical ozone advection and ozone photochemistry $(\alpha+$ term I + term II: dashed $)$ and radiative damping, vertical and meridional ozone advection, and ozone photochemistry $(\alpha+$ term I + term II + term III: dot-dash) for the Rossby-gravity wave using (a) zonal wind profile 1 and (b) zonal wind profile 2 . Terms I-III are defined in section 3.

of terms I and II are identical for the Kelvin and Rossbygravity waves; however, because the meridional motions are small $\left(v^{\prime} \approx 0\right)$ for the Kelvin wave, the heating due to meridional ozone advection (III) has negligible influence on its spatial modulation.

In the lower stratosphere (14) can be simplified by noting that $B$ and $C$ are small there, yielding an expression for the local spatial damping rate identical to that obtained in Echols and Nathan [1996; their Eq. (25)],

$$
m_{i, 0}=-\frac{m_{r, 0}}{2 \omega}\left[\alpha-\frac{A \bar{\gamma}_{z}}{N^{2}}\right] .
$$

Equation (15) is valid below $\sim 30 \mathrm{~km}$, where photochemical timescales are long compared to advective timescales. In this region $\left(\bar{\gamma}_{z}>0\right)$, vertical ozone advection leads to less wave damping. Above $35 \mathrm{~km}\left(\bar{\gamma}_{z}\right.$ $<0$ ), vertical ozone advection and ozone photochemistry lead to increased wave damping (equation not shown). The influence of ozone advection is maximized at $\sim 28 \mathrm{~km}$; that is, where $\bar{\gamma}_{z}$ is maximized.

\section{Influences of ozone heating on the zonal mean circulation \\ a. Eliassen-Palm flux}

To provide further insight into the influence of ozone feedbacks on the waves that affect the zonal mean circulation, we calculate the latitudinally integrated divergence of Eliassen-Palm flux, an important diagnostic quantity for assessing the influence of waves on the zonal mean circulation. Because the Kelvin and Rossbygravity waves are equatorially trapped, the meridional component of the latitudinally integrated EP flux vanishes, giving to lowest order,

$$
\langle\boldsymbol{\nabla} \cdot \mathbf{F}\rangle=\frac{\partial\left\langle F_{z}\right\rangle}{\partial z}=\frac{\partial}{\partial z}\left\langle\rho\left(\frac{R \beta y}{H N^{2}} \overline{v^{\prime} \mathrm{T}^{\prime}}-\overline{u^{\prime} w^{\prime}}\right)\right\rangle .
$$

The angle brackets represent a meridional integration, that is, \langle\rangle$=L^{-1} \int_{-\infty}^{\infty}() d y$, where $L=1200 \mathrm{~km}$ is a meridional scale of the waves. For adiabatic, inviscid flow, $\left\langle F_{z}\right\rangle$ is independent of height (Lindzen 1971). In contrast, when considering both radiative damping and ozone heating, we obtain

$$
\frac{\partial\left\langle F_{z}\right\rangle}{\partial z}=\sum_{j=0}^{1} A_{j}\left(z_{0}\right) m_{i, j} \exp \left[-2 \int_{z_{0}}^{z} m_{i, j} d z\right],
$$

where $A_{j}\left(z_{0}\right)$ represents the momentum flux at the lower boundary and $m_{i}$ represents the local spatial damping rate given by (14). Equation (17) therefore describes how NC and eddy ozone heating combine to modify the wave driving of the zonal mean flow.

To obtain quantitative results for ozone-induced changes in $\langle\boldsymbol{\nabla} \cdot \mathbf{F}\rangle,(17)$ is evaluated by choosing $A_{j}(17$ $\mathrm{km})= \pm 4 \times 10^{-3} \mathrm{~m}^{2} \mathrm{~s}^{-2}$ (HL) for various zonal wind profiles. We show the results using zonal wind profiles 1 and 2, which are qualitatively similar to the results obtained using other observed or analytical zonal wind profiles. We compare the influence of ozone feedbacks to an atmosphere without ozone feedbacks by setting the radiative-photochemical coefficients $A, B$, and $C$ equal to zero. Figures $7 \mathrm{a}$ and $7 \mathrm{~b}$ show a comparison of the normalized, latitudinally integrated EP flux divergence calculated with ozone (dashed lines) and without ozone (solid lines). Using zonal wind profile 1, the ozone-modified EP flux for the Kelvin wave $(c=30$ 

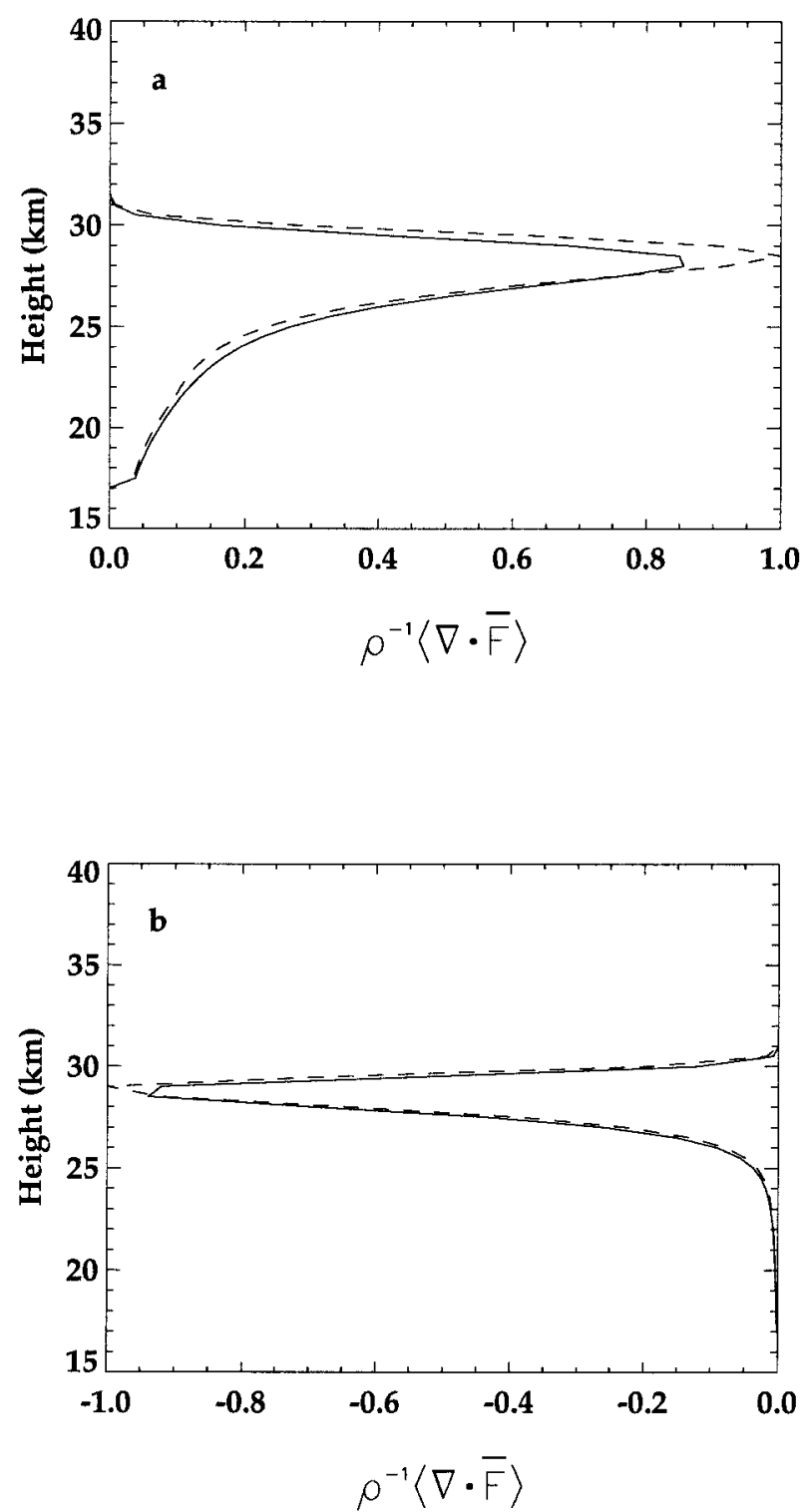

FIG. 7. Normalized latitudinally integrated divergence of EP flux for the (a) $s=1, c=30 \mathrm{~m} \mathrm{~s}^{-1}$ Kelvin wave using profile 1 and (b) $s=4, c=-30 \mathrm{~m} \mathrm{~s}^{-1}$ Rossby-gravity wave using profile 2 . Dashed lines indicate calculations including ozone feedbacks; solid lines indicate calculation without ozone feedbacks.

$\mathrm{m} \mathrm{s}^{-1}, s=1$ ) is larger above $\sim 29 \mathrm{~km}$, weaker below $\sim 29 \mathrm{~km}$, and has a maximum difference of $20 \%$ at 29 $\mathrm{km}$. Because Kelvin waves are preferentially absorbed in westerly shear zones (Andrews et al. 1987), $\langle\boldsymbol{\nabla} \cdot \mathbf{F}\rangle$ for profile 1 is positive (westerly acceleration) and has its peak coincident with the region of maximum wind shear $(\sim 29 \mathrm{~km})$. For zonal wind profile 2 (Fig 7b), $\langle\boldsymbol{\nabla} \cdot \mathbf{F}\rangle$ for the Rossby-gravity wave $\left(c=-30 \mathrm{~m} \mathrm{~s}^{-1}, s\right.$ $=4$ ) also is enhanced above $\sim 28 \mathrm{~km}$, reduced below $\sim 28 \mathrm{~km}$, and has a maximum difference of $7 \%$ at 28 $\mathrm{km}$. Because $m_{r}$ is proportional to $\omega^{-2}$ for the Rossbygravity wave and $\omega^{-1}$ for the Kelvin wave, the vertical profiles of EP flux for the Rossby-gravity wave are much sharper. Moreover, because $\omega$ depends more strongly on height for the Rossby-gravity wave, the influence of the ozone feedbacks is reduced.

\section{b. One-dimensional $Q B O$ model}

Quantitative results demonstrating how ozone feedbacks influence the QBO via modulation of the Kelvin and Rossby-gravity wave fields are obtained using a 1D model of the form (Holton and Lindzen 1972)

$$
\frac{\partial\langle\bar{u}\rangle}{\partial t}=-\frac{1}{\rho} \frac{\partial\left\langle F_{z}\right\rangle}{\partial z}+K \frac{\partial^{2} \bar{u}}{\partial z^{2}},
$$

where $\left\langle F_{z}\right\rangle$ is the latitudinally integrated vertical component of EP flux and $K$ is the eddy diffusion coefficient. Using an initial condition of zero zonal mean wind, (18) was integrated forward in time using a time step of 1 day, an eddy diffusion coefficient of $0.3 \mathrm{~m}^{2} \mathrm{~s}^{-1}$, and the same wave parameters as in section 3 (i.e., Kelvin wave: $c=30 \mathrm{~m} \mathrm{~s}^{-1}, s=1$; Rossby-gravity wave: $c=$ $\left.-30 \mathrm{~m} \mathrm{~s}^{-1}, s=4\right)$.

Figure 8 a shows a time-height cross section of the mean zonal wind for the reference case (no ozone feedbacks). The zonal mean wind oscillates with a period of about 27 months, has an amplitude range from +15 $\mathrm{m} \mathrm{s}^{-1}$ to $-16 \mathrm{~m} \mathrm{~s}^{-1}$, and each wind regime descends at a rate close to $1 \mathrm{~km} / \mathrm{mo}$. These results are qualitatively consistent with other 1D models of the QBO (e.g., HL; Dunkerton 1979). As shown in Fig. 8b, ozone-dynamics interactions produce stronger zonal winds $\left(1-2 \mathrm{~m} \mathrm{~s}^{-1}\right)$ and longer QBO periods ( $\sim 2$ months) when compared to the model run without ozone.

Results from the 1D model show that vertical ozone advection is destabilizing below $35 \mathrm{~km}$, resulting in enhanced wave driving of the zonal mean winds. In the 1D model, ozone feedbacks reduce the wave damping in the lower stratosphere. Weaker damping rates produce a slower decent of the shear zones (longer QBO periods) and a larger divergence of EP flux that drives a stronger zonal wind circulation (larger QBO amplitudes). Above $35 \mathrm{~km}$, the vertical extent of the QBO is limited by enhanced wave damping due to ozone photochemistry. The zonal wind profile is therefore in better agreement with observations of the equatorial stratosphere, which show a QBO signal from 17 to $35 \mathrm{~km}$ (Andrews et al. 1987). This underscores the importance of including eddy ozone feedbacks in bringing the model results in closer agreement with observations.

The above results indicate that the diabatic heating associated with ozone advection reduces the local spatial damping rates of the Kelvin and Rossby-gravity waves in the lower stratosphere. Because the ozone heating coefficient $A$ is still relatively small in the lower equatorial stratosphere, the waves remain damped with height. Nevertheless, this feedback significantly influences the zonal mean QBO. These results suggest that models of the QBO that include ozone-dynamics feed- 

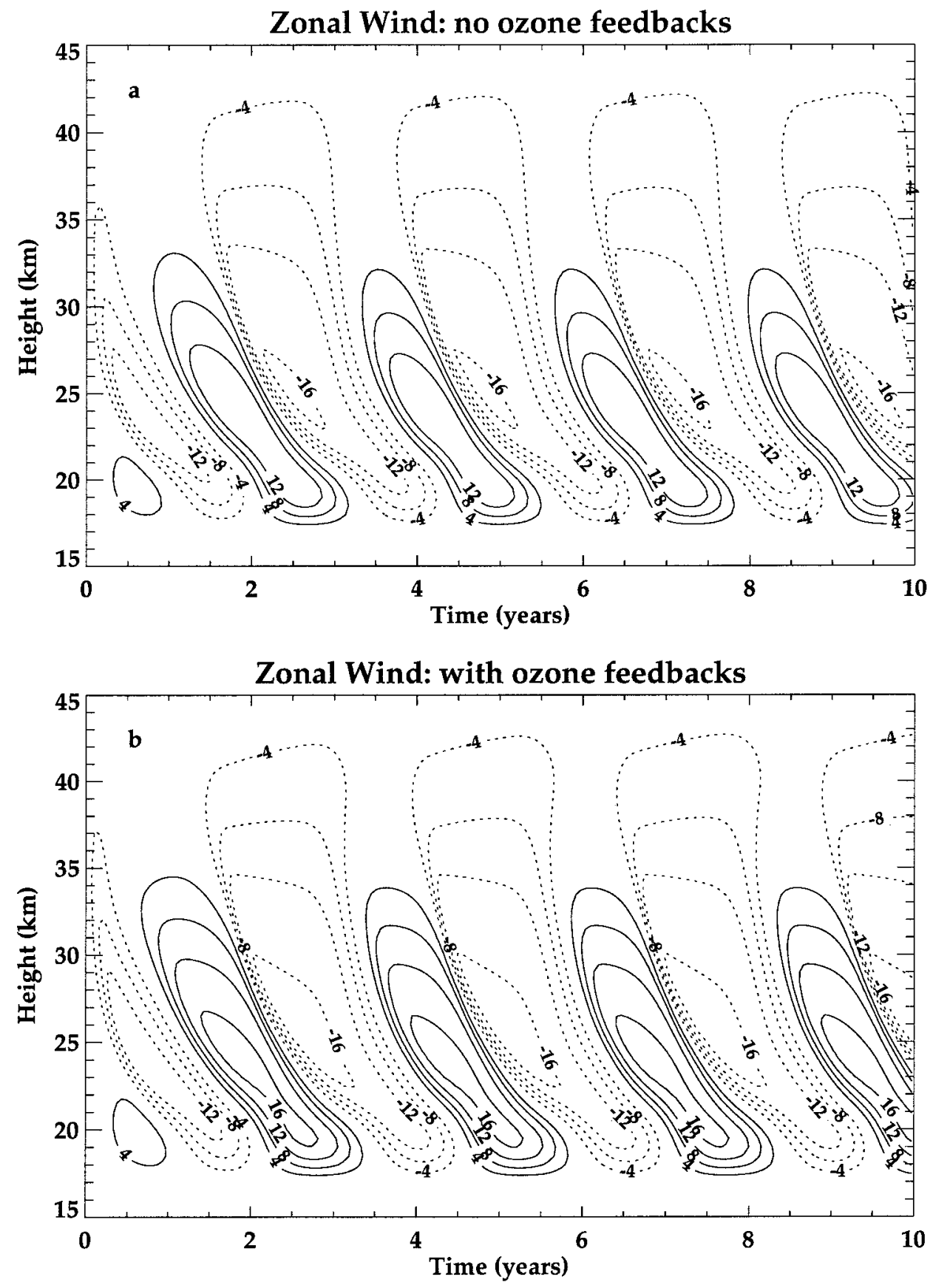

FIG. 8. Time-height section of zonal wind $\left(\mathrm{m} \mathrm{s}^{-1}\right)$ at the equator for (a) case without ozone feedbacks and (b) case including ozone feedbacks. Dotted contours indicate easterly winds.

backs would require smaller wave amplitudes at the lower boundary in order to obtain a QBO circulation.

\section{Summary and conclusions}

An equatorial beta-plane model is used to study the effects of ozone heating on the zonal wind QBO. Under the assumption of a slowly varying basic state and weak diabatic heating, we use a perturbation analysis to an- alytically demonstrate how the combined diabatic processes of ozone heating and radiative cooling modify the vertical (local) spatial damping rate of the Kelvin and Rossby-gravity waves. In the lower stratosphere, vertical ozone advection (spatially) destabilizes the waves, resulting in smaller $(15 \%)$ local damping rates from 22 to $30 \mathrm{~km}$; meridional ozone advection has a negligible influence at these levels of the stratosphere. Moreover, we have shown that the latitudinally inte- 
grated divergence of EP flux is altered by ozone feedbacks, resulting in a larger zonal mean driving force for both the Kelvin and Rossby-gravity waves.

The effects of the ozone-enhanced EP fluxes from the Kelvin and Rossby-gravity waves on the QBO are tested using a mechanistic 1D model of the QBO. Model integrations show that in the lower stratosphere ozone feedbacks increase the zonal wind amplitude by $1-2 \mathrm{~m}$ $\mathrm{s}^{-1}(10 \%-20 \%$ change) and increase the oscillation period by 2 months ( $10 \%$ change). In addition, the vertical extent of the QBO is found to be lower and in closer agreement with observations due to ozone photochemical damping. These results suggest the potential importance of eddy ozone feedbacks in simulating the QBO.

Although our results show that wave-ozone feedbacks affect the QBO by about $10 \%-20 \%$, these feedbacks are not sufficient to fully account for the apparent missing momentum in the QBO system. This is especially true for the Rossby-gravity wave, which has been observed to only have a fraction of the amplitude required to drive the easterly phase of the $\mathrm{QBO}$ (Boville and Randel 1992; Takahashi and Boville 1992). However, it is unclear how our results, which focus on $e d d y-$ ozone interactions, combined with the results of Hasebe (1994), which focus on zonal mean-ozone interactions, would influence the momentum budget of the zonal wind QBO. Clearly, both results, which explore dynam$\mathrm{ical} /$ photochemical feedbacks, are complementary, have similar potential relevance, and should be strongly considered in future work. Further understanding of these feedbacks requires the use of a more sophisticated model of the equatorial stratosphere capable of incorporating the explicit wave-mean flow interactions between the wave and zonal mean dynamical and ozone fields. Such work is currently under way and will be reported in a future paper.

Acknowledgments. The authors would like to thank the reviewers for their helpful comments and suggestions and Kristien King for assisting in the numerical calculations. This work was supported by the National Aeronautics and Space Administration (Grants NAG8871, NAG8-1054, NAG8-1143), the University of California Institute for Collaborative Research (INCOR), and Lawrence Livermore National Laboratory (Grants B291410, B291469). Support was also provided for Eugene Cordero through NASA's Global Change Fellowship Program (NASA Training Grant NGT-30153).

\section{APPENDIX A}

\section{Derivation of the Kelvin and Rossby-Gravity Wave Amplitude}

The perturbation analysis in section 3 hinges on two key assumptions: 1) that the diabatic heating rate due to ozone heating and radiative cooling is small and 2) that the background fields of wind, temperature, and ozone are slowly varying in the vertical. These assumptions are formalized by introducing the small parameter $\mu \ll 1$. In particular, we rescale the diabatic heating as $Q^{\prime} \Rightarrow \mu Q^{\prime}$ and introduce the long vertical scale $\zeta=\mu z$, which allows the vertical differential operator to be transformed as $\partial / \partial z \Rightarrow \partial / \partial z+\mu \partial / \partial \zeta$ (Andrews and McIntyre 1976). The basic-state fields of wind, temperature, and ozone are chosen of the form $\bar{u}$ $=\bar{u}(\zeta), \bar{T}=\bar{T}(y, \zeta), \bar{\gamma}=\bar{\gamma}(y, \zeta)$. We seek normalmode solutions of the form

$$
G^{\prime}(x, y, z, t)=\operatorname{Re}\left\{g(y, z) e^{z / 2 H} \operatorname{expi}(k x-\sigma t)\right\} .
$$

Equations (1)-(6) can then be expressed as

$$
\begin{aligned}
-i \omega u-\beta y v+i k \Phi & =-\mu w \bar{u}_{\zeta}, \\
-i \omega v+\beta y u+\Phi_{y} & =0, \\
i m \Phi-\frac{R T}{H} & =-\mu \Phi_{\zeta}, \\
i k u+v_{y}+i m w & =-\mu w_{\zeta}, \\
-i \omega T+\frac{H}{R} N^{2} w & =\frac{\mu H \beta y \bar{u}_{\zeta} v}{R}-\mu Q, \\
-i \omega \gamma+v \frac{\partial \bar{\gamma}}{\partial y}+w \frac{\partial \bar{\gamma}}{\partial z} & =-B \gamma-\frac{R}{H} C T,
\end{aligned}
$$

where $\omega$, the Doppler shifted frequency, is

$$
\omega=\omega(\zeta)=\sigma-k \bar{u}(\zeta),
$$

and subscripts denote partial differentiation. Because both the Kelvin and Rossby-gravity waves are characterized by relatively short vertical scales, terms involving the factor, $1 /(2 H)$, have been dropped, e.g., $m$ $\gg 1 /(2 H)$. Eliminating perturbation ozone from the diabatic heating term with the aid of (A7) gives

$$
Q=\frac{A H\left(v \bar{\gamma}_{y}+w \bar{\gamma}_{z}\right)}{R(B-i \omega)}+\left(\frac{A C}{B-i \omega}+\alpha\right) T .
$$

Each disturbance variable is expanded in a perturbation series in powers of $\mu$ :

$$
g=g_{0}+\mu g_{1}+\mu^{2} g_{2}+\cdots,
$$

where each term is assumed to have the normal mode form

$$
g_{r}(y, z)=\operatorname{Re}\left\{\hat{g}_{r}(y, \zeta) e^{i \mu^{-1} \phi(\zeta)}\right\},
$$

where

$$
m(\zeta)=d \phi / d \zeta .
$$

Equating the first two powers of $\mu$ we can express the equations in matrix form as

$$
\begin{aligned}
& \mathbf{M} \hat{\mathbf{U}}_{0}=0, \\
& \mathbf{M} \hat{\mathbf{U}}_{1}=A_{0},
\end{aligned}
$$

where 


$$
\mathbf{M}=\left(\begin{array}{ccccc}
\omega & -i \beta y & 0 & 0 & -k \\
i \beta y & \omega & 0 & 0 & i \partial / \partial y \\
0 & 0 & 0 & i N & m \\
0 & 0 & -i N & \omega & 0 \\
-k & i \partial / \partial y & m & 0 & 0
\end{array}\right)
$$

and

$\hat{\mathbf{U}}_{r}^{T}=\left\{\hat{u}_{r}, \hat{v}_{r},-\hat{w}_{r}, \frac{R}{H N} \hat{T}_{r}, \hat{\Phi}_{r}\right\}$,

$\mathbf{A}_{0}^{\mathrm{T}}=-i\left\{\bar{u}_{\zeta} w_{0}, 0,-\Phi_{0 \xi}, \frac{R}{H N} Q_{0}-\frac{\beta y \bar{u}_{\zeta}}{N} v_{0}, w_{0 \zeta}\right\} \cdot(\mathrm{A}$

The lowest-order equations yield the classic solutions for the equatorial Kelvin and Rossby-gravity waves: Kelvin wave:

$$
\begin{aligned}
& \left\{u_{0}, v_{0}, w_{0}, T_{0}, \Phi_{0}\right\} \\
& \quad=a_{\mathrm{K}}(\zeta)\left\{1,0, \frac{\omega}{N},-i \frac{H N}{R}, \frac{\omega}{k}\right\} \exp \left(\frac{-\beta\left|m_{K}\right| y^{2}}{2 N}\right) ;
\end{aligned}
$$

Rossby-gravity wave:

$$
\begin{aligned}
& \left\{u_{0}, v_{0}, w_{0}, T_{0}, \Phi_{0}\right\} \\
& =a_{\mathrm{RG}}(\zeta)\left\{\frac{i\left|m_{\mathrm{RG}}\right| \omega y}{N}, 1, \frac{i\left|m_{\mathrm{RG}}\right| \omega^{2} y}{N^{2}}, \frac{\left|m_{\mathrm{RG}}\right| H \omega y}{R}, i \omega y\right\} \\
& \quad \times \exp \left(\frac{-\beta\left|m_{\mathrm{RG}}\right| y^{2}}{2 N}\right) .
\end{aligned}
$$

The amplitudes $a_{\mathrm{K}}(\zeta)$ and $a_{\mathrm{RG}}(\zeta)$ are the remaining unknowns and are determined at $O(\mu)$. Because $\mathbf{M}$ is selfadjoint, a condition for the existence of higher-order solutions, $\hat{\mathbf{U}}_{1}$, yields (Andrews and McIntrye 1976)

$$
\left\{\mathbf{A}_{0}, \hat{\mathbf{U}}_{0}\right\}=0 .
$$

The inner product $\{$,$\} is defined as$

$$
\{a, b\}=\left\langle\sum_{n=1}^{5} a_{n} b_{n}^{*}\right\rangle,
$$

where

$$
\langle()\rangle=\int_{-\infty}^{\infty}() d y
$$

and the asterisk denotes complex conjugation. Substitution of (A15)-(A18) into (A19) and integrating in $y$ yields an ordinary differential equation for $a(\zeta)$; the solution is

$$
a_{j}(\zeta)=\frac{\text { const }}{|\omega|^{3 / 4}|\omega|^{3 j / 4}}[N(\beta+\omega k)]^{j / 4} \exp \left(i \int m_{j}(\zeta) d \zeta\right),
$$

where the local vertical wavenumber is defined as

$$
m_{j}(\zeta)=m_{r, j}(\zeta)+i m_{i, j}(\zeta)
$$

The subscript $j$ denotes the wave type $(j=0$ for the Kelvin wave; $j=1$ for the Rossby-gravity wave) and $r$ and $i$ denote real and imaginary components. The real component of the local vertical wavenumber, $m_{r}$, is approximated by the standard dispersion relationship for the Kelvin or Rossby-gravity wave (see appendix B), the imaginary component $m_{i}$ is

$$
\begin{aligned}
& m_{i, j} \\
& =-\frac{m_{r, j}}{2 \omega}\left[\alpha+\frac{1}{\left(B^{2}+\omega^{2}\right)}\right. \\
& \left.\times\{\underbrace{A B C}_{\mathrm{I}}-\underbrace{\frac{A \bar{\gamma}_{z} \omega^{2}}{N^{2}}}_{\mathrm{II}}-\delta_{j, 1} \underbrace{\frac{a A \bar{\gamma}_{y} B \omega}{N(\beta+\omega k)}}_{\text {III }}\}\right\},
\end{aligned}
$$

where $\delta_{j, 1}$ is the Kronecker delta function.

\section{APPENDIX B}

\section{Standard Dispersion Relationship for the Kelvin and Rossby-Gravity Wave}

The standard dispersion relationship for the Kelvin and Rossby-gravity is given, respectively, as

$$
\begin{aligned}
m_{r, 1} & =-\frac{N k}{\omega}, \\
\left|m_{r, 2}\right| & =\frac{N}{\omega^{2}}(\beta+\omega k) .
\end{aligned}
$$

\section{REFERENCES}

Andrews, D. G., and M. E. McIntyre, 1976: Planetary waves in horizontal and vertical shear: Asymptotic theory for equatorial waves in weak shear. J. Atmos. Sci., 33, 2049-2053.

—, J. R. Holton, and C. B. Leovy, 1987: Middle Atmosphere Dynamics. Academic Press, 489 pp.

Boville, B. A., and W. J. Randel, 1992: Equatorial waves in a stratospheric GCM: Effects of vertical resolution. J. Atmos. Sci., 49, 785-801.

Cogley, A. C., and W. J. Borucki, 1976: Exponential approximation for daily average solar heating or photolysis. J. Atmos. Sci., 33, 1347-1356.

Coy, L., 1979: An unusually large westerly amplitude of the quasibiennial oscillation. J. Atmos. Sci., 36, 174-176.

Dunkerton, T. J., 1979: On the role of the Kelvin wave in the westerly phase of the semiannual zonal wind oscillation. J. Atmos. Sci., 36, 32-41,

- 1985: A two-dimensional model of the quasi-biennial oscillation. J. Atmos. Sci., 42, 1151-1160.

Echols, R. S., and T. R. Nathan, 1996: Effects of ozone heating on forced equatorial Kevin waves. J. Atmos. Sci., 53, 263-275.

Fels, S. B., 1982: A parameterization of scale-dependent radiative damping rates in the middle atmosphere. J. Atmos. Sci., 39, 1141-1152.

Fleming, E. L., S. Chandra, M. R. Schoeberl, and J. J. Barnett, 1988: Monthly mean global climatology of temperature, wind, geo- 
potential height and pressure for $0-120 \mathrm{~km}$. NASA Tech. Memo. 100697, 85 pp. [Available from Eric Fleming, NASA/GSFC, Code 916, Greenbelt, MD 20771.]

Hartmann, D. L., and R. R. Garcia, 1979: A mechanistic model of ozone transport by planetary waves in the stratosphere. J. Atmos. Sci., 36, 350-364.

Hasebe, F., 1984: The global structure of the total ozone fluctuations observed on the time scales of two to several years. Dynamics of the Middle Atmosphere, J. R. Holton and T. Matsuno, Eds., Terra Scientific, 445-464.

—_ 1994: Quasi-biennial oscillations of ozone and diabatic circulation in the equatorial stratosphere. J. Atmos. Sci., 51, 729745.

Hayashi, Y., and D. G. Golder, 1994: Kelvin and mixed Rossbygravity waves appearing in the GDFL "SKYHI" general circulation model and the FGGE dataset: Implications for their generation mechanism and role in the QBO. J. Meteor. Soc. Japan, 72, 901-935.

Holton, J. R., and R. S. Lindzen, 1972: An updated theory for the quasi-biennial cycle of the tropical stratosphere. J. Atmos. Sci., 29, 1076-1080.

Keating, G. M., and D. F. Young, 1985: Interim reference ozone models for the middle atmosphere. Middle Atmosphere Program Handbook for MAP, Vol. 16, K. Labitzke, J. J. Barnett, and B. Edwards, Eds., University of Illinois, 318 pp.

Lindzen, R. S., 1971: Equatorial planetary waves in shear: Part I. J. Atmos. Sci., 28, 609-622.

— lation. J. Atmos. Sci., 25, 785-801.

Ling, X.-D., and J. London, 1986: The quasi-biennial oscillation of ozone in the tropical middle atmosphere: A one-dimensional model. J. Atmos. Sci., 43, 3122-3137.

McPeters, R. D., D. F. Heath, and P. K. Bhartia, 1984: Average ozone profiles for 1979 from the NIMBUS 7 SBUV instrument. $J$. Geophys. Res., 89, 5199-5214.

Nastrom, G. D., and A. D. Belmont, 1975: Periodic variation in stratospheric-mesospheric temperature from $20-65 \mathrm{~km}$ at $80^{\circ} \mathrm{N}$ to 30 S. J. Atmos. Sci., 32, 1715-1722.

Nathan, T. R., 1989: On the role of ozone in the stability of Rossby normal modes. J. Atmos. Sci., 46, 2094-2100.

_ , and L. Li, 1991: Linear stability of free planetary waves in the presence of radiative-photochemical feedbacks. J. Atmos. Sci., 48, 1837-1855.

— E. C. Cordero, and L. Li, 1994: Ozone heating and the destabilization of traveling waves during summer. Geophys. Res. Lett., 21, 1531-1534.

Pawson, S., R. S. Harwood, and J. D. Haigh, 1992: A study of the radiative dissipation of planetary waves using satellite data. $J$. Atmos. Sci., 49, 1304-1317.

Plumb, A. R., 1984: The quasi-biennial oscillation. Dynamics of the Middle Atmosphere, J. R. Holton and T. Matsuno, Eds., Terra Scientific, 217-251.

— , and R. C. Bell, 1982: A model of the quasi-biennial oscillation on an equatorial beta-plane. Quart. J. Roy. Meteor. Soc., 108, $335-352$.

Reed, R. J., 1964: A tentative model of the 26-month oscillation in tropical latitudes. Quart. J. Roy. Meteor. Soc., 90, 441-466.

— W. J. Campbell, L. A. Rasmussen, and D. G. Rogers, 1961: Evidence of downward-propagating annual wind reversal in the equatorial stratosphere. Geophys. Res. Lett., 4, 813-818.

Stolarski, R. S., and A. R. Douglass, 1985: Parameterization of the photochemistry of stratospheric ozone including catalytic loss processes. J. Geophys. Res., 90, 10 709-10 718.

Strobel, D., 1977: Photochemical-radiative damping and instability in the stratosphere. J. Geophys. Res., 82, 424-426.

— 1978: Parameterization of the atmospheric heating rate from 15 to $120 \mathrm{~km}$ due to $\mathrm{O}_{2}$ and $\mathrm{O}_{3}$ absorption of solar radiation. $J$. Geophys. Res., 83, 6225-6250.

Takahashi, M., 1987: A 2-dimensional numerical model of the quasibiennial oscillation: Part I. J. Meteor. Soc. Japan, 65, 523-536.

— 1996: Simulation of the stratospheric quasi-biennial oscillation using a general circulation model. Geophys. Res. Lett., 23, 661664.

— , and J. R. Holton, 1991: The mean zonal flow response to Rossby wave and gravity wave forcing in the equatorial lower stratosphere: Relationship to the QBO. J. Atmos. Sci., 48, 2078-2087.

— , and B. A. Boville, 1992: A three-dimensional simulation of the equatorial quasi-biennial oscillation. J. Atmos. Sci., 49, $1020-1035$.

Venyard, R. G., and R. A. Ebdon, 1961: Fluctuations in tropical stratospheric winds. Meteor. Mag., 90, 125-143.

Zhu, X., and J. R. Holton, 1986: Photochemical damping of inertiagravity waves. J. Atmos. Sci., 43, 2578-2584. 